\title{
Thickness-Dependent Beam Broadening in Transmission EBSD
}

\author{
Katherine P. Rice, Robert R. Keller \\ ${ }^{1}$ Applied Chemicals and Materials Division, National Institute of Standards and Technology, Boulder, \\ CO, USA.
}

Transmission electron backscatter diffraction (EBSD), also known as electron forward scatter diffraction or transmission Kikuchi diffraction in the SEM, can provide significant improvements in spatial resolution over conventional EBSD performed in reflection [1]. Remarkable results have been demonstrated, including phase identification of nanoparticles as small as $5 \mathrm{~nm}$ [2] and effective orientation mapping resolutions of $2 \mathrm{~nm}$ to $3 \mathrm{~nm}$ for thin metal films [3].

One of the primary factors to consider while discussing spatial resolution in the transmission configuration is where the most important diffraction occurs within the specimen thickness - this affects the achievable lateral resolution and determines which regions of the specimen give rise to diffraction patterns. We demonstrate through use of bilayer samples that the most important diffraction occurs within the bottom few nanometers of the crystalline region of a specimen. This means that the incident beam needs to traverse nearly the full thickness before undergoing detectable Kikuchi scattering. As this occurs, electron energies and trajectories change due to both elastic and inelastic scattering. These effects lead to beam broadening, and were modeled by Monte Carlo scattering simulations such as that shown in Figure 1 for $3000 \mathrm{~nm}$ of Al. The exit trajectories of all electrons are shown, which lead to estimates about effective beam size at the surface where detectable patterns are generated.

Estimates of the practically-achievable spatial resolution were made as a function of specimen massthickness by considering electron energy distributions associated with trajectories very near the exit surface. Figure 2 shows an example for varying thicknesses of copper. The result is a mass-thicknessdependent resolution that must be taken into account, particularly during orientation mapping.

*Contribution of NIST, not subject to copyright in the United States

\section{References:}

[1] Keller, RR; Geiss RH, Transmission EBSD from $10 \mathrm{~nm}$ domains in a scanning electron microscope. Journal of Microscopy 2012, 245 (3), 245-251.

[2] Brodusch, N.; Demers, H.; Gauvin, R., Nanometres-resolution Kikuchi patterns from materials science specimens with transmission electron forward scatter diffraction in the scanning electron microscope. Journal of Microscopy 2013, 250 (1), 1-14.

[3] Trimby, P. W., Orientation mapping of nanostructured materials using transmission Kikuchi diffraction in the scanning electron microscope. Ultramicroscopy 2012, 120 (0), 16-24. 


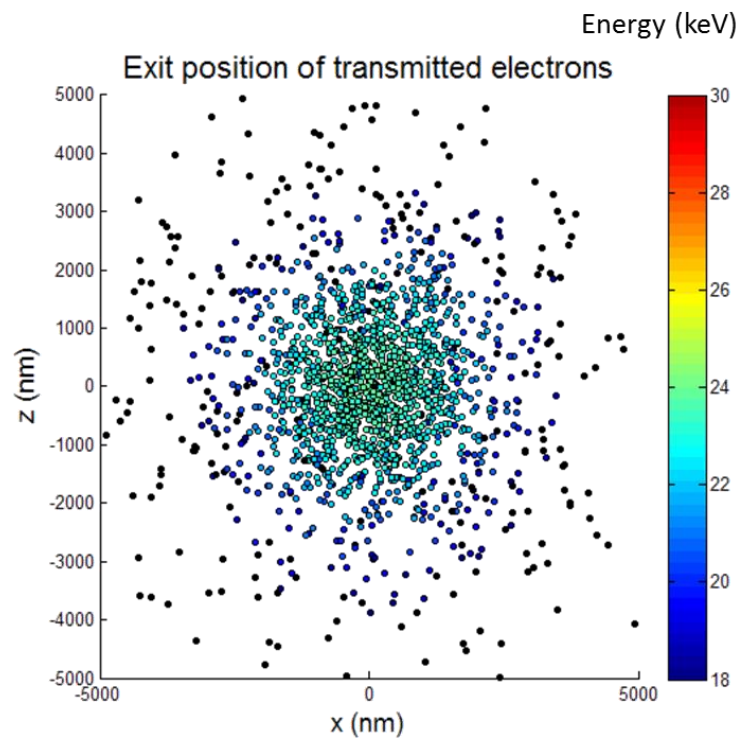

Exit position of transmitted electrons: $3000 \mathrm{~nm}$

Figure 1. Electron exit points from a $3000 \mathrm{~nm}$ Aluminum film at $30 \mathrm{keV}$, simulated by Monte Carlo methods.

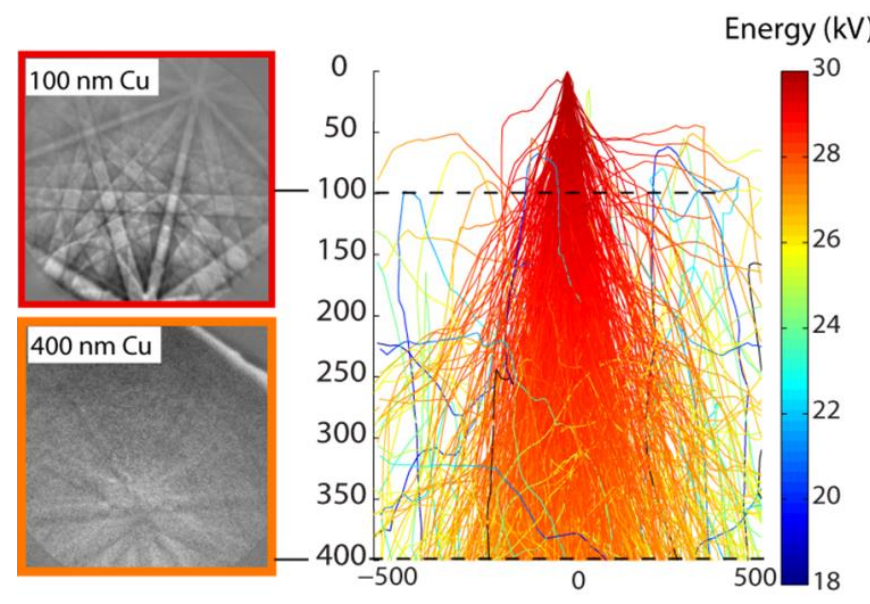

Figure 2. Kikuchi patterns from $100 \mathrm{~nm}$ and $400 \mathrm{~nm} \mathrm{Cu}$ films next to electron trajectory plots calculated by Monte Carlo methods. The trajectory color corresponds to the energy of the electrons at that point. Kikuchi patterns taken at an incident beam energy of $30 \mathrm{keV}$. 